\title{
Participação pública nos sistemas de saúde. Uma introdução
}

\section{Mauro Serapioni}

\section{(2) OpenEdition}

\section{Journals}

Edição electrónica

URL: http://journals.openedition.org/rccs/8264

DOI: $10.4000 /$ rccs.8264

ISSN: 2182-7435

Editora

Centro de Estudos Sociais da Universidade de Coimbra

\section{Edição impressa}

Data de publição: 1 dezembro 2018

Paginação: 91-98

ISSN: 0254-1106

\section{Refêrencia eletrónica}

Mauro Serapioni, «Participação pública nos sistemas de saúde. Uma introdução », Revista Crítica de Ciências Sociais [Online], 117 | 2018, posto online no dia 03 dezembro 2018, consultado o 24 setembro 2020. URL : http://journals.openedition.org/rccs/8264 ; DOI : https://doi.org/10.4000/rccs.8264

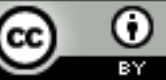




\section{MAURO SERAPIONI}

\section{Participação pública nos sistemas de saúde. Uma introdução*}

As inovações democráticas que envolvem os/as cidadãos/ãs nos processos de decisão política têm assumido grande relevância a nível internacional, tendo-se multiplicadas, nos últimos anos, as iniciativas de participação promovidas tanto pelas instituições públicas como pela sociedade civil (Santos, 2003; Smith, 2010; Pateman, 2012). Este debate tem vindo a ser intensificado nas ciências sociais aplicadas à saúde. Vários estudos realçam a acentuada dificuldade de inter-relação entre, por um lado, a tecnicização da medicina e a organização burocrática do sistema de saúde e, por outro, o padrão de comunicação mais natural do "mundo da vida", segundo a aceção de Habermas (Barry et al., 2001; Chaudhary et al., 2013).

É, portanto, manifesta a necessidade de promover uma maior autonomia dos pacientes e uma relação mais equitativa com os profissionais. Existe também uma crescente procura de melhor qualidade na atenção à saúde, uma maior personalização e humanização dos cuidados a prestar e uma valorização da voz dos/as cidadãos/ãs. A literatura assinala diversos argumentos a favor da participação pública nos sistemas de saúde: valoriza o saber e a experiência dos/as pacientes, melhorando, à partida, a qualidade das decisões, conduzindo, consequentemente, a melhores resultados de saúde (van de Bovenkamp et al., 2009); contribui para o incremento da responsabilidade e da transparência nos serviços de saúde (Tritter e McCallum, 2006); aumenta o empoderamento dos/as pacientes (Wallerstein, 2006); fortalece as atividades de promoção da saúde (Serapioni e Matos, 2013); reforça a representatividade dos grupos mais vulneráveis (Timotijevic e Raats, 2007). Potenciar a perspetiva dos/as cidadãos/ãs representa também uma importante estratégia para superar o crescente "défice democrático" que caracteriza os sistemas

* Este texto foi desenvolvido com o apoio concedido pela Fundação para a Ciência e a Tecnologia (SFRH/BPD/98655/2013). 
de saúde (Cooper et al., 1995; Coulter, 2013). Neste contexto, várias organizações internacionais (WHO, 2015; OECD, 2005; Council of Europe, 2001) têm incentivado os governos nacionais a desenvolverem espaços públicos dentro da sociedade civil como locus adequados para democratizar os sistemas de saúde.

Até ao momento, foram desenvolvidas várias iniciativas e mecanismos para envolver os/as cidadãos/ãs - conselhos de saúde no Brasil, em Espanha e em Portugal, comités consultivos mistos na Itália, júris de cidadãos/ãs em Inglaterra e nos Estados Unidos da América, ChoiceWork Dialogue no Canadá, etc. (Degeling et al., 2015; Serapioni e Matos, 2016) - e investidos recursos significativos no desenho e implementação de tais práticas participativas. Entretanto, os resultados de diversas investigações (Morrison e Dearde, 2013; Conklin et al., 2015) indicam que, apesar das boas intenções e de alguns esforços louváveis empreendidos, o grau de institucionalização da participação ainda está longe de ser satisfatório. A maioria dos benefícios esperados da participação pública baseia-se, principalmente, em pressupostos teóricos e normativos, e não tanto em dados empíricos (Contandriopoulos, 2004; Boivin et al., 2014).

Neste prisma, diversos estudos têm apontado os pontos críticos que afetam a qualidade e a efetividade da participação pública nos sistemas de saúde. Alguns dos mais significativos são os seguintes: 1) a falta de representatividade dos fóruns participativos e sua dificuldade em incluir os diversos segmentos da sociedade civil, nomeadamente os grupos socialmente excluídos (Contandriopoulos, 2004; Li et al., 2015); 2) a necessidade de criar mais mecanismos capazes de produzir decisões coletivas e de aumentar a legitimidade das decisões. Neste âmbito, os métodos deliberativos têm despertado um grande interesse no campo da saúde, em virtude de sua dinâmica organizativa inovadora, na qual os/as participantes podem expressar livremente os seus argumentos, num ambiente democrático (Avritzer, 2011; Fung, 2015); 3) a escassez de investigações avaliativas que demonstrem a efetividade das práticas de participação pública (Burton, 2009; Serapioni, 2014). Apesar de anos de experiências e práticas participativas, ainda poucos estudos se debruçaram sobre a forma como os/as cidadãos/ãs poderão ser legítima e efetivamente envolvidos/as nas decisões sobre políticas e organização do cuidado em saúde (Boivin et al., 2014; Conklin et al., 2015).

Os contributos incluídos neste dossier debruçam-se sobre algumas das dimensões críticas da participação assinaladas nesta introdução. São de salientar, aqui, três artigos (do Brasil, Espanha e Itália) que abordam os dispositivos de participação promovidos pelas instituições públicas, 
regionais ou estaduais, também denominados como "espaços convidados" (invited spaces) (Cornwall e Coelho, 2006; Gaventa, 2006). O quarto artigo descreve as iniciativas promovidas pelas associações de doentes em Portugal (created spaces), que reivindicam a abertura de novos fóruns e canais de participação a nível local, regional e nacional (claimed spaces) (Cornwall e Coelho, 2006; Gaventa, 2006). Os fóruns de participação da Europa do Sul abordados neste dossier (Espanha, Itália e Portugal) desempenham um papel consultivo, ao passo que a experiência do Brasil é deliberativa, no sentido em que os/as representantes escolhidos/as participam diretamente nos processos de tomada de decisão.

No texto "Avanços e desafios da participação institucionalizada no sistema de saúde do Brasil”, José Patrício Bispo Júnior faz uma descrição dos principais dispositivos de participação institucionalizada, no âmbito do Sistema Único de Saúde do Brasil, nomeadamente as conferências e os conselhos de saúde. Para além disso, analisa os resultados de um estudo de caso sobre o Conselho de Saúde do estado da Bahia. O autor realça os avanços do conselho - no que diz respeito à maior inclusão e ampliação das entidades sociais envolvidas no processo de participação - assinalando, ao mesmo tempo, o escasso poder de decisão (ou "de agenda") dos/as representantes dos/as utentes e dos/as profissionais. $\mathrm{O}$ que pode ser interpretado como um indicador de resistência dos/as gestores/as em reequilibrar as relações assimétricas de poder entre os/ /as diferentes protagonistas bem como constatar um nível insuficiente de influência do conselho sobre a definição de políticas de saúde do estado.

Marta Aguilar Gil e José María Bleda García analisam em "El modelo de participación ciudadana en salud en Puertollano (España): más allá de la voluntad política y del empoderamiento ciudadano" os fatores que influenciaram o desaparecimento de uma inovadora experiência de participação implementada na área de saúde de Puertollano, na Comunidade Autónoma de Castilla-La Mancha, em Espanha. Este modelo de participação - composto por um fórum participativo de saúde, um conselho de participação e administração bem como um fórum virtual - funcionou regularmente entre 2008 e 2011, envolvendo um número crescente de associações de pacientes e de cidadãos/ãs. Entretanto, o governo conservador que ganhou as eleições regionais de 2011 não deu continuidade à experiência participativa que, em poucos anos, foi desmantelada, sem que houvesse quaisquer formas de resistência da sociedade civil, dos/as profissionais de saúde e dos partidos políticos da oposição. Entre os fatores responsáveis pela interrupção prematura 
desta experiência, os autores identificam a falta de um real envolvimento das instituições políticas, a sedimentação insuficiente do projeto na cultura das associações de saúde e a escassa formação de agentes envolvidos/as sobre o tema da participação.

$\mathrm{O}$ artigo de Silvia Cervia, intitulado "Citizen Engagement and the Challenge of Democratizing Health: An Italian Case Study", analisa o processo de reorganização da governança da região Toscana (Itália) que, a partir de 2008, instituiu 20 consórcios de saúde (Società della Salute - SDS) para responder às necessidades sociais e de saúde da população. Como parte essencial deste processo, em cada SDS foram introduzidos comités de participação, compostos por representantes das comunidades locais, e grupos consultivos, incluindo organizações de voluntariado e do terceiro setor que trabalham no campo da saúde e dos assuntos sociais. Os resultados dos estudos de caso destacam algumas experiências bem-sucedidas, particularmente no caso dos SDS que estabeleceram regras claras de funcionamento e asseguraram um apoio organizacional às instâncias representativas das comunidades locais, estendendo, desta forma, os seus papéis e influência. Entretanto, como consequência da crise financeira, em 2010, a reorganização regional foi fortemente travada pelo governo nacional, situação que gerou um enfraquecimento desta restruturação.

Em "Participação pública na saúde: das ideias à ação em Portugal”, Sofia Crisóstomo e Margarida Santos apresentam a experiência de ativismo em saúde desenvolvida por uma rede de associações de doentes e utentes com o objetivo de institucionalizar mecanismos participativos, no âmbito do Serviço Nacional de Saúde português. As autoras, após terem assinalado o desfasamento entre o envolvimento público - reconhecido tanto na Constituição como nos planos nacionais de saúde - e a ausência de canais de participação efetivos, descrevem as estratégias e as atividades realizadas no âmbito do projeto "MAIS PARTICIPAÇÃO melhor saúde" (MPms). Adotando abordagens participativas e metodologias de aprendizagem baseadas na investigação-ação, o projeto MPms conseguiu obter resultados relevantes, como: o levantamento de perceções e experiências sobre o tema da participação, tanto de doentes e utentes como das associações que os representam; a elaboração, discussão pública e lançamento da petição "Carta para a participação pública em saúde"; e a entrega dessa petição à Assembleia da República e aos/às deputados/as da Comissão Parlamentar de Saúde.

Os resultados dos casos examinados neste dossier contribuem assim para ampliar o conhecimento sobre as dimensões e os pontos críticos 
da participação pública nos sistemas de saúde, assim como identificar os fatores contextuais que influenciam a efetividade dos processos participativos e os potenciais défices de representatividade.

\section{Referências bibliográficas}

Avritzer, Leonardo (2011), "Introdução. A qualidade da democracia e a questão da efetividade da participação: mapeando o debate", in Roberto Rocha C. Pires (org.), Diálogos para o desenvolvimento - Efetividade das instituições participativas no Brasil: estratégias de avaliação, 7. Brasília: IPEA - Instituto de Pesquisa Econômica Aplicada, 13-28.

Barry, Christine A.; Stevenson, Fiona; Britten, Nicky; Barber, Nick; Bradley, Colin (2001), "Giving Voice to the Lifeworld. More Human, More Effective Medical Care? A Qualitative Study of Doctor-Patient Communication in General Practice", Social Science \& Medicine, 53(4), 478-505.

Burton, Paul (2009), "Conceptual, Theoretical and Practical Issues in Measuring the Benefits of Public Participation", Evaluation, 15(3), 263-284.

Boivin, Antoine; Lehoux, Pascale; Burgers, Jackio; Grol, Richard (2014), "What Are the Key Ingredients for Effective Public Involvement in Health Care Improvement and Policy Decisions? A Randomized Trial Process Evaluation", The Milbank Quarterly, 92(2), 319-350.

Chaudhary, Sarah; Avis, Mark; Munn-Giddings, Carol (2013), "Beyond the Therapeutic: A Habermasian View of Self-Help Groups' Place in the Public Sphere", Social Theory \& Health, 11(1), 59-80.

Conklin, Annalijn; Morris, Zoë; Nolte, Ellen (2015), "What is the Evidence Base for Public Involvement in Health-Care Policy? Results of a Systematic Scoping Review", Health Expectations, 18(2), 153-165.

Contandriopoulos, Damien (2004), "Sociological Perspective on Public Participation in Health Care", Social Science \& Medicine, 58, 321-330.

Cooper, Liz; Coote, Ann; Davis, Ann; Jackson, Christine (1995), Voices Off: Tackling the Democratic Deficit in Health. London: IPPR - Institute for Public Policy Research.

Cornwall, Andrea; Coelho, Vera (orgs.) (2006), Spaces for Change? The Politics of Citizen Participation in New Democratic Arenas. London: Zed Books.

Coulter, Angela (2013), Engaging Patients in Healthcare. New York: McGraw Hill Professional.

Council of Europe, Committee of Ministers (2001), The Development of Structures for Citizen and Patient Participation in the Decision-Making Process Affecting Health Care: Recommendation Rec (2000) and Explanatory Memorandum. Strasbourg: Council of Europe Publishing. 
Degeling, Chris; Carter, Stacy M.; Rychetnik, Lucie (2015), "Which Public and why Deliberate? A Scoping Review of Public Deliberation in Public Health and Health Policy Research”, Social Science \& Medicine, 131, 114-121.

Fung, Archon (2015), "Putting the Public Back into Governance: The Challenges of Citizen Participation and Its Future”, Public Administration Review, 75(4), 513-522.

Gaventa, John (2006), "Finding the Spaces for Change: A Power Analysis", IDS Bulletin, 37(6), 23-33.

Li, Kathy; Abelson, Julia; Giacomini, Mita; Contandriopoulos, Damien (2015), "Conceptualizing the Use of Public Involvement in Health Policy Decision-Making”, Social Science \& Medicine, 138, 14-21.

Morrison, Cecily; Dearden, Andy (2013), “Beyond Tokenistic Participation: Using Representational Artefacts to Enable Meaningful Public Participation in Health Service”, Health Policy, 112, 179-186.

OECD - Organisation for Economic Co-operation and Development (2005), Evaluating Public Participation in Policy-Making. Paris: OECD.

Pateman, Carol (2012), "Participatory Democracy Revisited”, Perspect on Politics, 10(1), 7-19.

Santos, Boaventura de Sousa (2003), Democratizar a democracia: os caminhos da democracia participativa. Porto: Edições Afrontamento.

Serapioni, Mauro (2014), "Os desafios da participação e da cidadania nos sistemas de saúde”, Ciência \& Saúde Coletiva, 19(12), 4829-4839.

Serapioni, Mauro; Matos, Ana Raquel (2013), "Participação em saúde: entre limites e desafios, rumos estratégias”, Revista Portuguesa de Saúde Pública, 31(1), 11-22.

Serapioni, Mauro; Matos, Ana Raquel (orgs.) (2016), "Participação em saúde: experiências brasileiras e do Sul da Europa - A modo de apresentação”, O Público e o Privado, 27, 11-16.

Smith, Graham (2010), Democratic Innovations: Designing Institutions for Citizen Participation. Cambridge: Cambridge University Press.

Timotijevic, Lada; Raats, Monique (2007), "Evaluation of Two Methods of Deliberative Participation of Older People in Food-Policy Development”, Health Policy, 82(3), 302-319.

Tritter, Jonathan Q.; McCallum, Alison (2006), “The Snakes and Ladders of User Involvement: Moving beyond Arnstein”, Health Policy, 76, 156-158.

van de Bovenkamp, Hester M.; Trappenburg, Margo J.; Grit, Kor J. (2009), "Patient Participation in Collective Healthcare Decision Making: The Dutch Model”, Health Expect, 13(1), 73-85.

WHO - World Health Organization (2015), Taking a Participatory Approach to Development and Better Health. Copenhagen: WHO - Regional Office for Europe. 
Participação pública nos sistemas de saúde. Uma introdução | 97

Wallerstein, Nina (2006), What is the Evidence on Effectiveness of Empowerment to Improve Health? Copenhagen: World Health Organization - Regional Office for Europe.

\section{Mauro Serapioni}

Centro de Estudos Sociais da Universidade de Coimbra

Colégio de S. Jerónimo, Largo D. Dinis, Apartado 3087, 3000-995 Coimbra, Portugal

Contacto: mauroserapioni@ces.uc.pt 
